\title{
Robotic surgery is the optimal approach for mitral surgery
}

\author{
Christopher Cao ${ }^{1,2}$, Ashleigh L. Clark ${ }^{1}$, Rakesh M. Suri ${ }^{3}$ \\ ${ }^{1}$ Collaborative Research Group, Macquarie University, Sydney, Australia; ${ }^{2}$ University of New South Wales, Sydney, Australia; ${ }^{3}$ Department of \\ Thoracic and Cardiovascular Surgery, Cleveland Clinic Foundation, Cleveland, USA \\ Correspondence to: Rakesh M. Suri, MD, DPhil. Department of Thoracic and Cardiovascular Surgery, Cleveland Clinic Foundation, 9500 Euclid Ave \\ J4-1, Cleveland, OH 44195, USA. Email: surir@ccf.org.
}

\begin{abstract}
This report presented an overview of the patient selection, technical considerations and clinical evidence for robotic mitral valve surgery. A review of comparative outcomes to medical therapy, sternotomy approach, and the MitraClip device suggested that robotic mitral valve surgery is safe and effective in specialized centres. Potential benefits include a reliable and durable repair, with reduced perioperative morbidity and improved quality of life. Future studies should aim to delineate mid- and long-term clinical and echocardiographic outcomes following robotic mitral valve repair compared to the conventional sternotomy approach.
\end{abstract}

Keywords: Mitral valve; robotic; repair

Submitted Mar 29, 2016. Accepted for publication Oct 12, 2016.

doi: 10.21037/acs.2016.10.01

View this article at: http://dx.doi.org/10.21037/acs.2016.10.01

Robotic mitral valve surgery has been demonstrated as a safe and effective approach for selected patients in specialized centres (1-3). Mitral valve repair for patients with myxomatous disease has been successfully performed through standardized robotic techniques with excellent clinical outcomes (4). It is imperative to emphasize that the primary objective of robotic surgery in such patients is identical to mitral repair through any surgical approach-to reliably achieve a complete and durable repair with minimal residual regurgitation. Recent studies have recommended surgery be performed in a timely manner for patients with severe mitral regurgitation, even without class I triggers such as heart failure and left ventricular dysfunction $(5,6)$. The previous 'watchful waiting' strategy should no longer be considered 'benign' as it has been shown to be associated with worse long-term mortality and heart failure outcomes compared to patients who undergo earlier surgery in specialized centres (7). We hereby present an overview of the technical aspects of robotic mitral valve surgery, current evidence on clinical and cost-related outcomes and address potential areas of improvement and future research.

\section{Patient selection and technical considerations}

As with all surgical procedures, patient selection is critical, and robotic surgery is not appropriate for all patients with severe mitral regurgitation (8). Those with significant coronary artery disease, peripheral vascular disease and previous right thoracotomy are generally not ideal candidates for a robotic approach. In addition, patients with severe mitral annular calcification should be excluded from robotic surgery due to absent tactile feedback in the current robotic devices. Preoperative imaging investigations include routine transthoracic echocardiogram, as well as ECG-gated computerized tomographic angiography of the chest, abdomen and pelvis to assess coronary and peripheral arteries.

Details of our standardized surgical techniques have been described previously (4). Briefly, a camera port is placed $2-\mathrm{cm}$ lateral to the right nipple in the $4^{\text {th }}$ intercostal space, followed by a $1.5-2 \mathrm{~cm}$ working port laterally and two $0.8 \mathrm{~cm}$ ports above and below the working port. A left atrial retractor port is placed $3-4 \mathrm{~cm}$ medial to the camera port in the $4^{\text {th }}$ intercostal space. The pericardium is then opened anterior to the phrenic nerve and suspended by stay sutures pulled through the chest wall. After $350 \mathrm{IU} / \mathrm{kg}$ of intravenous heparin, we perform right femoral arterial, femoral and right internal jugular venous cannulation for cardiopulmonary bypass, after confirming that the activated clotting time is $>450$ seconds. Cardioplegia is given through 
an ascending aortic vent cannula after the application of a transthoracic clamp. After cardioplegia is achieved, we gain access to the mitral valve through an incision posterior to the interatrial groove. The appropriate surgical technique is then selected based on the patient's mitral valve pathology, emulating exactly the techniques we would utilise via open median sternotomy.

\section{Safety and efficacy}

In the absence of randomized controlled trials, it is important to consider the direct comparative outcomes of existing therapeutic modalities, as emphasized by the National Heart, Lung and Blood Institute (9).

\section{Early mitral repair versus medical management}

The Mitral Regurgitation Internal Database assessed 2,097 patients with flail mitral regurgitation from six international tertiary centres to compare early surgical intervention with 'watchful waiting' involving medical therapy and echocardiographic monitoring. Of the 1,021 patients who did not present with Class I triggers of heart failure or left ventricular dysfunction, 446 patients underwent mitral valve surgery within three months of diagnosis and 575 patients underwent initial medical management. After a mean follow-up of 10.3 years with $98 \%$ complete followup, it was demonstrated that patients who underwent early surgery had similar short-term mortality and heart failure outcomes compared to medical therapy, but significantly improved survival ( $86 \%$ vs. $69 \%$ at 10 years, $\mathrm{P}<0.001)$ and heart failure ( $7 \%$ vs. $23 \%$ at 10 years, $\mathrm{P}<0.001)$ outcomes at long-term follow-up. These findings were confirmed with risk-adjusted models, propensity score matched comparisons and inverse probability-weighted analysis. This large multi-institutional study provided vital data, propelling a paradigm shift in the management of patients with severe degenerative mitral valve regurgitation in the contemporary clinical setting, to favour earlier intervention (6). Similarly, other studies reaffirmed that severe uncorrected mitral regurgitation has deleterious clinical outcomes and performing mitral valve surgery within 3 months of diagnosis results in significant improvements in long-term survival and freedom from heart failure $(7,10)$.

\section{Robotic versus sternotomy mitral valve repair}

A recent systematic review and meta-analysis identified six relevant studies that compared surgery for degenerative mitral valve disease through the robotic $(n=960)$ versus sternotomy ( $n=690)$ approach (11). Meta-analysis of the available data demonstrated significantly lower mortality ( $0.5 \%$ vs. $2.2 \%, \mathrm{P}=0.02)$ and similar stroke $(0.8 \%$ vs. $2.4 \%$, $\mathrm{P}=0.54$ ) outcomes for the robotic approach. Although patients who underwent robotic surgery had slightly longer cardiopulmonary bypass and cross-clamp time, the duration of intensive care stay and hospitalization were not significantly different. Both surgical approaches were associated with short- to mid-term efficacy with satisfactory echocardiographic outcomes, with more than $90 \%$ of patients having no or trivial residual regurgitation. It is important to recognize that all six studies included in this meta-analysis were retrospective in nature and baseline patient characteristics differed between the treatment arms. Nonetheless, it can be shown that robotic mitral valve surgery can be safely performed by expert surgeons for selected patients with favourable clinical outcomes.

\section{Mitral valve surgery versus Mitraclip}

There is a relative paucity of robust clinical data on the percutaneous MitraClip device (Abbott Vascular, Santa Clara, CA, USA) beyond short-term follow-up. A systematic review identified 12 prospective studies with very limited data beyond 1 -year, at which time survival ranged between $72-100 \%$ (12). Outcomes of a meta-analysis to compare mitral valve surgery with Mitraclip were difficult to interpret, as patients differed in valvular pathology and other important baseline patient characteristics. However, it was clear that MitraClip was associated with significantly higher incidences of $>2$ residual mitral regurgitation compared to surgery $(17.2 \%$ vs. $0.4 \%$, $\mathrm{P}<0.0001)$ (13). Judging by these studies, further evidence on mid- and long-term outcomes are required for the Mitraclip device prior to routine use in the clinical setting.

\section{Cost and quality of life}

Although there are considerable costs related to the current da Vinci Surgical System (Intuitive Surgical, Sunnyvale, CA, USA) and its maintenance, significant savings may be achieved through earlier postoperative recovery and reduced duration of hospitalization. A recent analysis of the National Inpatient Sample identified 631 pairs of propensity score matched patients who underwent mitral valve repair through the robotic or sternotomy approach (14). Patients who underwent robotic surgery were associated with a 
significantly shorter duration of hospitalization (4 vs. 6 days, $\mathrm{P}<0.001)$ and no significant differences in total costs were identified. This was consistent with institutional studies that reported similar costs of robotic versus conventional mitral valve surgery (15). We have also demonstrated that cost savings can be made through systems innovation to optimize in-hospital resource utilization (16).

To assess the quality of life of patients after mitral valve surgery, we conducted a prospective study involving 202 consecutive patients who underwent robotic surgery or sternotomy (17). Using a range of recognized measurement tools such as the Duke Activity Status Index, the Short Form 12-Item Health Survey, and Single-item, Linear Analogue Self-Assessment scales, we found that robotic repair was associated with improved outcomes at one year. In addition, patients who underwent robotic surgery were able to return to work significantly earlier than those who underwent sternotomy (median 33 vs. 54 days, $\mathrm{P}<0.001$ ). Other experienced groups also demonstrated that robotic mitral valve repair is associated with shorter hospital length of stay, quicker return to normal activities, and a superior cosmetic result $(7,18,19)$.

Robotic mitral valve repair requires a thoughtful prospective and individualised plan for cardiopulmonary bypass and myocardial protection, particularly in patients with more than mild aortic regurgitation. However, excellent arterial perfusion and venous drainage can be obtained by femoral artery cannulation and advancing a cannula from the femoral vein into the superior vena cava, respectively. Furthermore, an antegrade cardioplegia cannula placed in the proximal ascending aorta and/or a retrograde cardioplegia catheter placed via the internal jugular vein enables excellent myocardial protection with standard cardioplegia solutions. However, in cases with challenging coronary sinus access, use of a 'single shot' cardioplegia solution (e.g., Del Nido cardioplegia) simplifies myocardial protection.

Severe mitral annular calcification can be an additional concern when performing robotic mitral valve repair due to the fact that the robot is not equipped with the calcium removal tool. However, for patients with focal calcification of mitral annulus that can be repaired without calcium debridement, robotic mitral valve repair is feasible. In summary, careful patient selection is necessary, however, all categories of mitral leaflet prolapse may be repaired with $99 \%$ certainty (1).

\section{Conclusions}

In conclusion, existing evidence suggests that robotic mitral valve surgery is safe and effective in specialized centres. Potential benefits include a very high likelihood of valve repair, mortality risk $<0.2 \%$, reduced perioperative morbidity and improved quality of life. Latest midterm outcome data have confirmed that the results are durable following robotic mitral valve repair. These benefits may facilitate earlier referral of patients with severe mitral regurgitation with Class IIa triggers for surgery. As demonstrated previously, these asymptomatic patients should be offered earlier repair in specialty heart valve practices to prevent symptoms and restore normal life expectancy. Ultimately, the best mitral valve repair procedure for a patient is the approach that offers a complete and durable repair, irrespective of the size or location of the incision. Due to the fact that the same technical operation can be performed using robotic technology and high definition visualization, patients benefit from minimally invasive robotic approaches to mitral valve repair. Future studies should focus on further delineating mid- and long-term clinical and echocardiographic outcomes following robotic mitral valve repair.

\section{Acknowledgements}

None.

\section{Footnote}

Conflicts of Interest: The authors have no conflicts of interest to declare.

\section{References}

1. Suri RM, Taggarse A, Burkhart HM, et al. Robotic Mitral Valve Repair for Simple and Complex Degenerative Disease: Midterm Clinical and Echocardiographic Quality Outcomes. Circulation 2015;132:1961-8.

2. Mihaljevic T, Jarrett CM, Gillinov AM, et al. Robotic repair of posterior mitral valve prolapse versus conventional approaches: potential realized. J Thorac Cardiovasc Surg 2011;141:72-80.e1-4.

3. Chitwood WR Jr, Rodriguez E, Chu MW, et al. Robotic mitral valve repairs in 300 patients: a single-center experience. J Thorac Cardiovasc Surg 2008;136:436-41.

4. Suri RM, Burkhart HM. Optimizing outcomes of robotic mitral valve repair for all prolapse anatomy: the SuriBurkhart technique. Ann Cardiothorac Surg 2013;2:841-5.

5. Nishimura RA, Otto CM, Bonow RO, et al. 2014 AHA/ 
ACC Guideline for the Management of Patients With Valvular Heart Disease: executive summary: a report of the American College of Cardiology/American Heart Association Task Force on Practice Guidelines. Circulation 2014;129:2440-92.

6. Suri RM, Schaff HV, Enriquez-Sarano M. Mitral valve repair in asymptomatic patients with severe mitral regurgitation: pushing past the tipping point. Semin Thorac Cardiovasc Surg 2014;26:95-101.

7. Suri RM, Vanoverschelde JL, Grigioni F, et al. Association between early surgical intervention vs watchful waiting and outcomes for mitral regurgitation due to flail mitral valve leaflets. JAMA 2013;310:609-16.

8. Suri RM, Dearani JA, Mihaljevic T, et al. Mitral valve repair using robotic technology: Safe, effective, and durable. J Thorac Cardiovasc Surg 2016;151:1450-4.

9. Hlatky MA, Douglas PS, Cook NL, et al. Future directions for cardiovascular disease comparative effectiveness research: report of a workshop sponsored by the National Heart, Lung, and Blood Institute. J wendAm Coll Cardiol 2012;60:569-80.

10. Suri RM, Burkhart HM, Rehfeldt KH, et al. Robotic mitral valve repair for all categories of leaflet prolapse: improving patient appeal and advancing standard of care. Mayo Clin Proc 2011;86:838-44.

11. Cao C, Wolfenden H, Liou K, et al. A meta-analysis of robotic vs. conventional mitral valve surgery. Ann Cardiothorac Surg 2015;4:305-14.

Cite this article as: Cao C, Clark AL, Suri RM. Robotic surgery is the optimal approach for mitral surgery. Ann Cardiothorac Surg 2016;5(6):563-566. doi: 10.21037/ acs.2016.10.01
12. Munkholm-Larsen S, Wan B, Tian DH, et al. A systematic review on the safety and efficacy of percutaneous edge-to-edge mitral valve repair with the MitraClip system for high surgical risk candidates. Heart 2014;100:473-8.

13. Wan B, Rahnavardi M, Tian DH, et al. A meta-analysis of MitraClip system versus surgery for treatment of severe mitral regurgitation. Ann Cardiothorac Surg 2013;2:683-92.

14. Paul S, Isaacs AJ, Jalbert J, et al. A population-based analysis of robotic-assisted mitral valve repair. Ann Thorac Surg 2015;99:1546-53.

15. Kam JK, Cooray SD, Kam JK, et al. A cost-analysis study of robotic versus conventional mitral valve repair. Heart Lung Circ 2010;19:413-8.

16. Suri RM, Thompson JE, Burkhart HM, et al. Improving affordability through innovation in the surgical treatment of mitral valve disease. Mayo Clin Proc 2013;88:1075-84.

17. Suri RM, Antiel RM, Burkhart HM, et al. Quality of life after early mitral valve repair using conventional and robotic approaches. Ann Thorac Surg 2012;93:761-9.

18. Paul S, Isaacs AJ, Jalbert J, et al. A population-based analysis of robotic-assisted mitral valve repair. Ann Thorac Surg 2015;99:1546-53.

19. Ramzy D, Trento A, Cheng $W$, et al. Three hundred robotic-assisted mitral valve repairs: the Cedars-Sinai experience. J Thorac Cardiovasc Surg 2014;147:228-35. 